\title{
Application of the Dahl friction model in the dynamics analysis of grab cranes
}

\author{
A. Urbaś
}

University of Bielsko-Biala, Department of Mechanics, 43-309 Bielsko-Biala, Willowa 2, Poland

\begin{abstract}
The paper presents dynamics analysis of grab cranes. The cranes can be built out of any number of links, however, only selected links have their own drive that is considered in the flexible form. The cranes are mounted to the ground by means of any number of flexible supports. Joint coordinates and homogeneous transformation matrices are used to describe the cranes' geometry. Equations of motion are derived using Lagrange equations. Friction phenomenon is taken into account in all joints. The joint forces and torques used to calculate friction torques in the revolute joints and friction forces in the prismatic joints are determined using the recursive Newton-Euler algorithm. Models of revolute and prismatic joints are worked out. Friction coefficients are defined using the Dahl friction model. The results of numerical calculations present the influence of friction on the driving torques, force and behaviour of the load.
\end{abstract}

\section{Nomenclature}

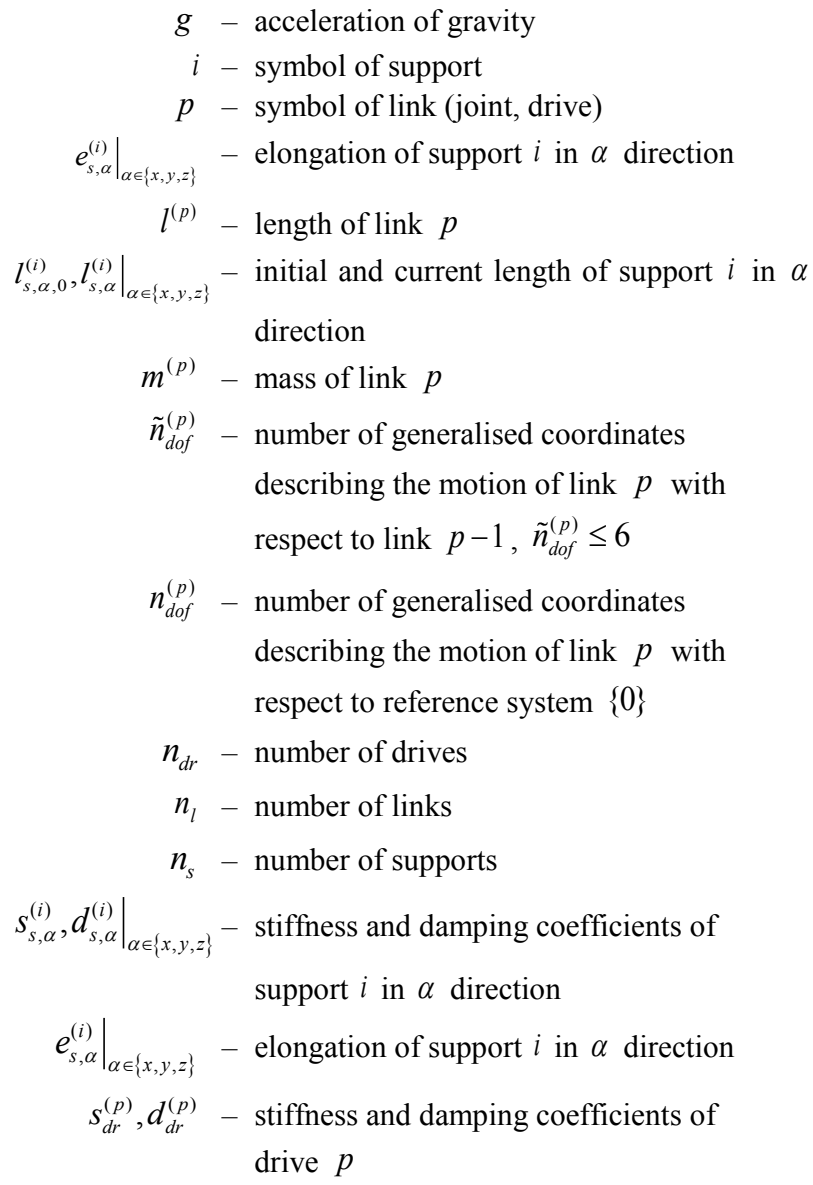
drive $p$

$\tilde{\mathbf{q}}^{(p)}-$ vector of generalised coordinates describing the motion of link $p$ with respect to link $p-1$

$\mathbf{q}^{(p)}$ - vector of generalised coordinates describing the motion of link $p$ with respect to reference system $\{0\}$

$\mathbf{r}_{C^{(p)}}^{(p)}-$ vector of position of point $C^{(p)}$ defined in the local coordinate system of link $p$

$\mathbf{H}^{(p)}$ - pseudo-inertia matrix of link $p$

$\tilde{\mathbf{T}}^{(p)}$ - homogeneous transformation matrix from the local coordinate system of link $p$ to the system of link $p-1$

$\mathbf{T}^{(p)}$ - homogeneous transformation matrix from the local coordinate system of link $p$ to reference system $\{0\}$,

$$
\mathbf{T}_{i}^{(p)}=\frac{\partial \mathbf{T}^{(p)}}{\partial q_{i}^{(p)}}, \mathbf{T}_{i, j}^{(p)}=\frac{\partial^{2} \mathbf{T}^{(p)}}{\partial q_{i}^{(p)} \partial q_{j}^{(p)}}
$$

Friction parameters

$$
\begin{aligned}
i- & \text { parameter that defines the shape of the } \\
& \text { material curve } \\
\left.\mu_{\alpha}^{(p)}\right|_{\alpha \in\{A, B, C\}}, \mu^{(p)}- & \text { friction coefficient in the revolute and } \\
& \text { prismatic joint } \\
\left.\mu_{\alpha, 0}^{(p)}\right|_{\alpha \in\{A, B, C\}}, \mu_{0}^{(p)}- & \text { maximum value of the friction coefficient } \\
& \text { in the revolute and prismatic joint } \\
\left.\sigma_{\alpha}^{(p)}\right|_{\alpha \in\{A, B, C\}}, \sigma^{(p)}- & \text { contact stiffness coefficient of the } \\
& \text { revolute and prismatic joint }
\end{aligned}
$$




\section{Introduction}

Grab cranes belong to the group of handling equipment that is used in various industries, and in particular in the forest industry. A versatile analysis of these cranes is conducted by means of computer methods of modelling and simulation. For this purpose, engineers use commercial programs or, less often, authors' programs which are cheaper and dedicated for specific applications. However, the essential advantage of creating their own programs is primarily the ability to test alternative and sometimes new methods of modelling (e.g. flexibility of links, friction) or to solve equations of motion.

The mathematical model for dynamics analysis of a grab crane [1-4] built with eight links is presented in the paper. Flexibility of the support system and drives is taken into account. The influence of friction in the joints on the driving torques and force is analysed.

\section{Mathematical model of the grab crane}

As an example, the dynamics analysis of a grab crane built out of eight links, of which only five have their own drive with driving torque/force $\left.\mathbf{t}_{d r}^{(p)}\right|_{p=2,3,4,5,8}$ (Fig. 1), is presented. The crane is sited by means of eight flexible supports.

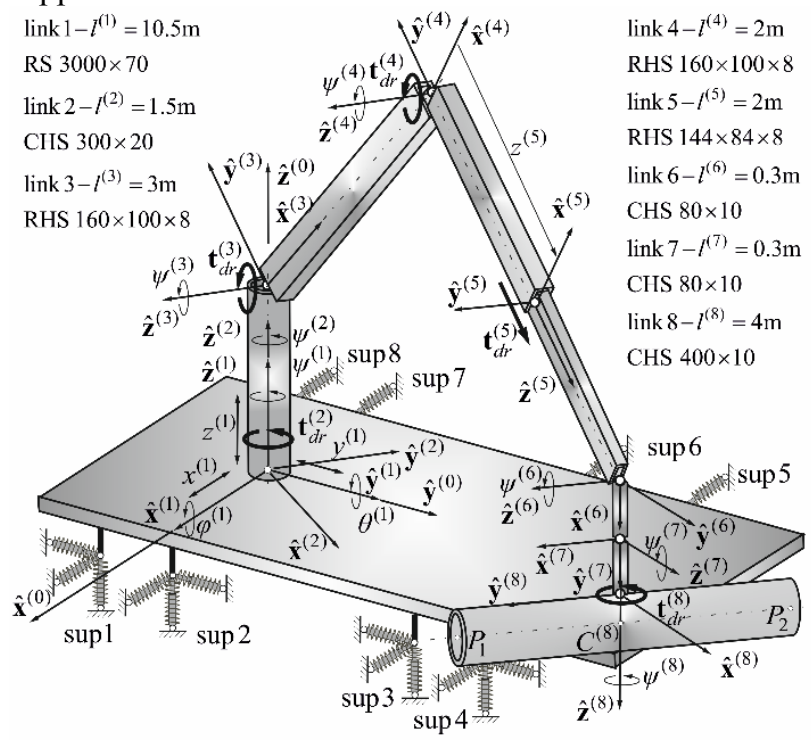

Fig. 1. Model of the grab crane.

The motion of link $p$ of the crane is described by the vector of joint coordinates:

$$
\left.\mathbf{q}^{(p)}\right|_{p=1, \ldots, n_{l}}=\left[\begin{array}{ll}
\mathbf{q}^{(p-1)^{T}} & \tilde{\mathbf{q}}^{(p)^{T}}
\end{array}\right]^{T}
$$

where: $\mathbf{q}^{(0)}=\varnothing$,

$$
\begin{aligned}
& \tilde{\mathbf{q}}^{(1)}=\left[\begin{array}{llllll}
x^{(1)} & y^{(1)} & z^{(1)} & \psi^{(1)} & \theta^{(1)} & \varphi^{(1)}
\end{array}\right]^{T}, \tilde{\mathbf{q}}^{(2)}=\left[\begin{array}{l}
\psi^{(2)}
\end{array}\right], \\
& \tilde{\mathbf{q}}^{(3)}=\left[\psi^{(3)}\right], \tilde{\mathbf{q}}^{(4)}=\left[\psi^{(4)}\right], \tilde{\mathbf{q}}^{(5)}=\left[z^{(5)}\right], \tilde{\mathbf{q}}^{(6)}=\left[\psi^{(6)}\right], \\
& \tilde{\mathbf{q}}^{(7)}=\left[\psi^{(7)}\right], \tilde{\mathbf{q}}^{(8)}=\left[\psi^{(8)}\right] \text {. }
\end{aligned}
$$

The Denavit-Hartenberg notation [5] is used to describe the geometry of the crane. The homogeneous transformation matrices from the local coordinate systems to the reference system are determined as follows:

$$
\left.\mathbf{T}^{(p)}\right|_{p=1, \ldots, n_{l}}=\mathbf{T}^{(p-1)} \tilde{\mathbf{T}}^{(p)}
$$

where: $\mathbf{T}^{(0)}=\mathbf{I}$,

$$
\begin{aligned}
& \tilde{\mathbf{T}}^{(1)}=\left[\begin{array}{cc}
\mathrm{c} \psi^{(1)} \mathrm{c} \theta^{(1)} & \mathrm{c} \psi^{(1)} \mathrm{s} \theta^{(1)} \mathrm{s} \varphi^{(1)}-\mathrm{s} \psi^{(1)} \mathrm{c} \varphi^{(1)} \\
\mathrm{s} \psi^{(1)} \mathrm{c} \theta^{(1)} & \mathrm{s} \psi^{(1)} \mathrm{s} \theta^{(1)} \mathrm{s} \varphi^{(1)}+\mathrm{c} \psi^{(1)} \mathrm{c} \varphi^{(1)} \\
-\mathrm{s} \theta^{(1)} & \mathrm{c} \theta^{(1)} \mathrm{s} \varphi^{(1)} \\
0 & 0
\end{array}\right. \\
& \left.\begin{array}{cc}
\mathrm{c} \psi^{(1)} \mathrm{s} \theta^{(1)} \mathrm{c} \varphi^{(1)}+\mathrm{s} \psi^{(1)} \mathrm{s} \varphi^{(1)} & x^{(1)} \\
\mathrm{s} \psi^{(1)} \mathrm{s} \theta^{(1)} \mathrm{c} \varphi^{(1)}-\mathrm{c} \psi^{(1)} \mathrm{s} \varphi^{(1)} & y^{(1)} \\
\mathrm{c} \theta^{(1)} \mathrm{c} \varphi^{(1)} & z^{(1)} \\
0 & 1
\end{array}\right] \text { - nonlinear model, }
\end{aligned}
$$$$
\tilde{\mathbf{T}}^{(1)}=\left[\begin{array}{cccc}
1 & -\psi^{(1)} & \theta^{(1)} & x^{(1)} \\
\psi^{(1)} & 1 & -\varphi^{(1)} & y^{(1)} \\
-\theta^{(1)} & \varphi^{(1)} & 1 & z^{(1)} \\
0 & 0 & 0 & 1
\end{array}\right] \text { - linear model, }
$$$$
\begin{aligned}
\tilde{\mathbf{T}}^{(2)} & =\left[\begin{array}{cccc}
\mathrm{c} \psi^{(2)} & -\mathrm{s} \psi^{(2)} & 0 & 0 \\
\mathrm{~s} \psi^{(2)} & \mathrm{c} \psi^{(2)} & 0 & 0 \\
0 & 0 & 1 & 0 \\
0 & 0 & 0 & 1
\end{array}\right], \\
\tilde{\mathbf{T}}^{(3)} & =\left[\begin{array}{cccc}
\mathrm{c} \psi^{(3)} & -\mathrm{s} \psi^{(3)} & 0 & 0 \\
0 & 0 & -1 & 0 \\
\mathrm{~s} \psi^{(3)} & \mathrm{c} \psi^{(3)} & 0 & l^{(2)} \\
0 & 0 & 0 & 1
\end{array}\right],
\end{aligned}
$$$$
\tilde{\mathbf{T}}^{(4)}=\left[\begin{array}{cccc}
\mathrm{c} \psi^{(4)} & -\mathrm{s} \psi^{(4)} & 0 & l^{(3)} \\
\mathrm{s} \psi^{(4)} & \mathrm{c} \psi^{(4)} & 0 & 0 \\
0 & 0 & 1 & 0 \\
0 & 0 & 0 & 1
\end{array}\right]
$$$$
\tilde{\mathbf{T}}^{(5)}=\left[\begin{array}{cccc}
1 & 0 & 0 & 0 \\
0 & 0 & -1 & -z^{(5)} \\
0 & 1 & 0 & 0 \\
0 & 0 & 0 & 1
\end{array}\right]
$$$$
\tilde{\mathbf{T}}^{(6)}=\left[\begin{array}{cccc}
\mathrm{c} \psi^{(6)} & -\mathrm{s} \psi^{(6)} & 0 & 0 \\
0 & 0 & 1 & 0 \\
-\mathrm{s} \psi^{(6)} & -\mathrm{c} \psi^{(6)} & 0 & l^{(5)} \\
0 & 0 & 0 & 1
\end{array}\right] \text {, }
$$$$
\tilde{\mathbf{T}}^{(8)}=\left[\begin{array}{cccc}
\mathrm{c} \psi^{(8)} & -\mathrm{s} \psi^{(8)} & 0 & 0 \\
0 & 0 & 1 & l^{(7)} \\
-\mathrm{s} \psi^{(8)} & -\mathrm{c} \psi^{(8)} & 0 & 0 \\
0 & 0 & 0 & 1
\end{array}\right] \text {. }
$$

In order to determine friction torques in the revolute joints and friction forces in the prismatic joints, models of these joints were developed (Fig. 2). 


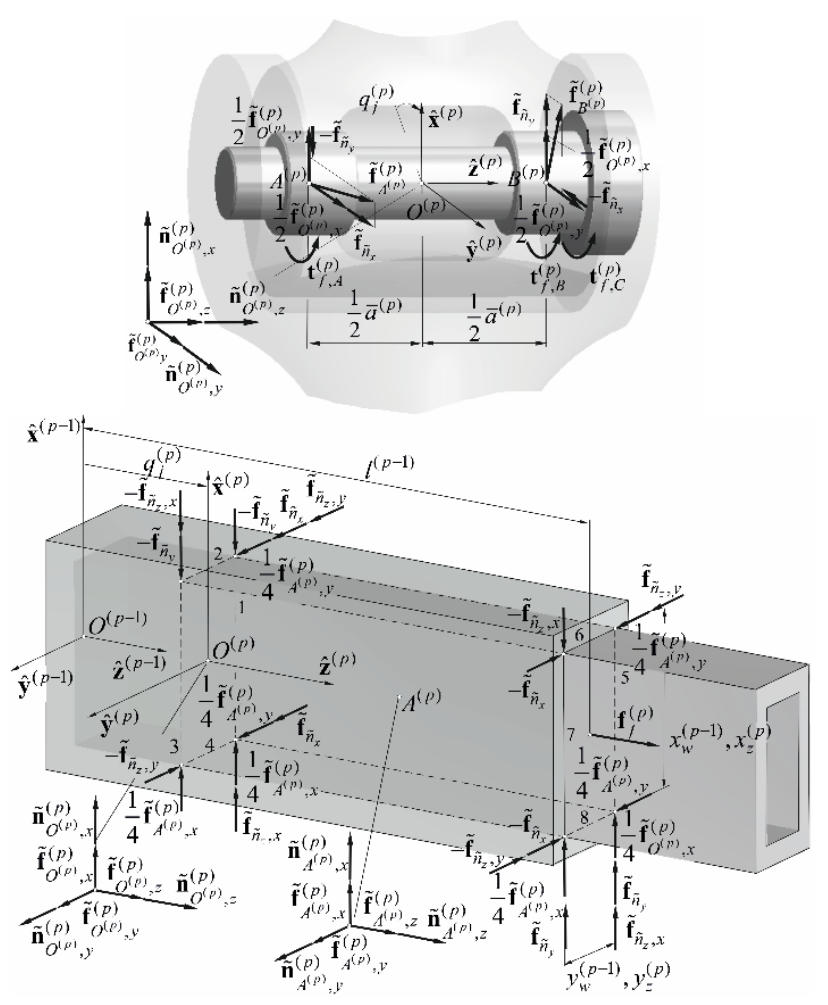

Fig. 2. Models of revolute and prismatic joints.

In order to determine the joint forces $\tilde{\mathbf{f}}_{o^{(p)}}^{(p)}$ and torques $\tilde{\mathbf{n}}_{O^{(p)}}^{(p)}$, and then the values of friction torques and forces, the recursive Newton-Euler algorithm [5] is used.

The values of the friction coefficients are defined using the Dahl friction model [6], described by the following form:

$$
\begin{aligned}
& \left.\dot{\mu}\right|_{\substack{\dot{\mu}=\dot{\mu}_{\alpha}^{(p)} \\
\dot{\mu}=\dot{\mu}^{(p)}}}=\sigma \dot{q}_{j}^{(p)}\left(1-\frac{\mu}{\mu_{0}} \operatorname{sgn}\left(\dot{q}_{j}^{(p)}\right)\right)^{i} \times \\
& \times\left.\operatorname{sgn}\left(1-\frac{\mu}{\mu_{0}} \operatorname{sgn}\left(\dot{q}_{j}^{(p)}\right)\right)\right|_{\substack{\mu_{0}=\mu_{\alpha, 0}^{(p)}, \sigma=\sigma_{\alpha}^{(p)} \\
\mu_{0}=\mu_{0}^{(p)}, \sigma=\sigma^{(p)}}}
\end{aligned}
$$

The equations of motion are derived using the Lagrange equations and the algorithms taken from paper [7]:

$$
\mathbf{A} \ddot{\mathbf{q}}=\mathbf{e}+\mathbf{f}_{s}+\mathbf{t}_{d r}-\mathbf{s}_{f},
$$

where: $\quad \mathbf{A}=\left[\begin{array}{ccccc}\mathbf{A}_{1,1} & \cdots & \mathbf{A}_{1, j} & \cdots & \mathbf{A}_{1, n_{l}} \\ \vdots & & \vdots & & \vdots \\ \mathbf{A}_{i, 1} & \cdots & \mathbf{A}_{i, j} & \cdots & \mathbf{A}_{i, n_{l}} \\ \vdots & & \vdots & & \vdots \\ \mathbf{A}_{n_{l}, 1} & \cdots & \mathbf{A}_{n_{l}, j} & \cdots & \mathbf{A}_{n_{l}, n_{l}}\end{array}\right]$,

$$
\begin{aligned}
& \mathbf{A}_{i, j}=\sum_{l=\max \{i, j\}}^{n_{l}} \tilde{\mathbf{A}}_{i, j}^{(l)},\left.\tilde{\mathbf{A}}_{i, j}^{(p)}\right|_{i, j=1, \ldots, p}=\left(\tilde{a}_{n_{d o f}^{(i-1)}+k, n_{d o f}^{(j-1)}+l}^{(j)}\right)_{\substack{\left.k=1, \ldots, \tilde{n}_{d o j}^{(i)} \\
l=1, \ldots, \tilde{n}_{d o f}\right)}}, \\
& \tilde{a}_{i, j}^{(p)}=\operatorname{tr}\left\{\mathbf{T}_{i}^{(p)} \mathbf{H}^{(p)} \mathbf{T}_{j}^{(p)^{T}}\right\}
\end{aligned}
$$

$$
\begin{aligned}
& \mathbf{e}=\left[\begin{array}{c}
\mathbf{e}_{1} \\
\vdots \\
\mathbf{e}_{i} \\
\vdots \\
\mathbf{e}_{n_{l}}
\end{array}\right], \mathbf{e}_{i}=-\sum_{l=i}^{n_{l}}\left(\tilde{\mathbf{h}}_{i}^{(l)}+\tilde{\mathbf{g}}_{i}^{(l)}\right), \\
& \left.\tilde{\mathbf{h}}_{i}^{(p)}\right|_{i=1, \ldots, p}=\left(\tilde{h}_{n_{d o f}^{(i-1)}+k}^{(p)}\right)_{k=1, \ldots, \tilde{n}_{d o f}^{(p)}}, \\
& \tilde{h}_{i}^{(p)}=\sum_{m=1}^{n_{\text {dof }}^{(p)}} \sum_{n=m}^{n_{\text {dof }}^{(p)}} \operatorname{tr}\left\{\mathbf{T}_{i}^{(p)} \mathbf{H}^{(p)} \mathbf{T}_{m, n}^{(p)}\right\} \dot{q}_{m}^{(p)} \dot{q}_{n}^{(p)}, \\
& \left.\tilde{\mathbf{g}}_{i}^{(p)}\right|_{i=1, \ldots, p}=\left(\tilde{g}_{n_{d o f}^{(i-1)}+k}^{(p)}\right)_{k=1, \ldots, \tilde{n}_{d o f}^{(i)}}, \tilde{g}_{i}^{(p)}=m^{(p)} g \mathbf{j}_{3} \mathbf{T}_{i}^{(p)} \tilde{\mathbf{r}}_{C^{(p)}}^{(p)}, \\
& \mathbf{j}_{3}=\left[\begin{array}{llll}
0 & 0 & 1 & 0
\end{array}\right] \text {, } \\
& \mathbf{f}_{s}=\left[-\left(\frac{\partial E_{p, s}}{\partial \tilde{\mathbf{q}}^{(1)}}+\frac{\partial R_{s}}{\partial \dot{\tilde{\mathbf{q}}}^{(1)}}\right)^{T} \quad \mathbf{0}\right]^{T}, \\
& E_{p, s}=\frac{1}{2} \sum_{i=1}^{n_{s}} \sum_{\alpha \in\{x, y, z\}} s_{s, \alpha}^{(i)}\left(e_{s, \alpha}^{(i)}\right)^{2},
\end{aligned}
$$$$
R_{s}=\frac{1}{2} \sum_{i=1}^{n_{s}} \sum_{\alpha \in\{x, y, z\}} d_{s, \alpha}^{(i)}\left(\dot{e}_{s, \alpha}^{(i)}\right)^{2}
$$$$
\mathbf{t}_{d r}=\left[\begin{array}{llllllll}
\mathbf{0} & t_{d r}^{(2)} & t_{d r}^{(3)} & t_{d r}^{(4)} & t_{d r}^{(5)} & 0 & 0 & t_{d r}^{(8)}
\end{array}\right]^{T},
$$$$
t_{d r}^{(p)}=-\left(\frac{\partial E_{p, d r}^{(p)}}{\partial q_{j}^{(p)}}+\frac{\partial R_{d r}^{(p)}}{\partial \dot{q}_{j}^{(p)}}\right),
$$$$
E_{p, d r}^{(p)}=\frac{1}{2} s_{d r}^{(p)}\left(q_{d r}^{(p)}-q_{j}^{(p)}\right)^{2}, R_{d r}^{(p)}=\frac{1}{2} d_{d r}^{(p)}\left(\dot{q}_{d r}^{(p)}-\dot{q}_{j}^{(p)}\right)^{2},
$$$$
\mathbf{s}_{f}=\left[\begin{array}{llllllll}
\mathbf{0} & t_{f}^{(2)} & t_{f}^{(3)} & t_{f}^{(4)} & f_{f}^{(5)} & t_{f}^{(6)} & t_{f}^{(7)} & t_{f}^{(8)}
\end{array}\right]^{T},
$$$$
t_{f}^{(p)}=t_{f, A}^{(p)}+t_{f, B}^{(p)}+t_{f, C}^{(p)},
$$$$
t_{f, A}^{(p)}=\frac{1}{2} \mu_{A}^{(p)} \tilde{f}_{A^{(p)}}^{(p)} d_{A}^{(p)}, t_{f, B}^{(p)}=\frac{1}{2} \mu_{B}^{(c, p)} \tilde{f}_{B^{(p)}}^{(p)} d_{B}^{(p)},
$$$$
t_{f, C}^{(p)}=\frac{1}{3} \mu_{C}^{(p)}\left|\tilde{f}_{O^{(p)}, z}^{(p)}\right| \frac{d_{C}^{(p)^{3}}-d_{A}^{(p)^{3}}}{d_{C}^{(p)^{2}}-d_{A}^{(p)^{2}}},
$$$$
f_{f}^{(p)}=\mu^{(p)} \tilde{f}^{(p)}, \tilde{f}^{(p)}=\sum_{i=1}^{8} \tilde{f}_{i}^{(p)} .
$$

The equations of motion are integrated using the RungeKutta method of the fourth order with a fixed step equal to $10^{-4}$. 


\section{Results of the calculations}

The influence of friction on the courses of the driving torques in the revolute joints and driving force in the prismatic joint are presented in Fig. 4.

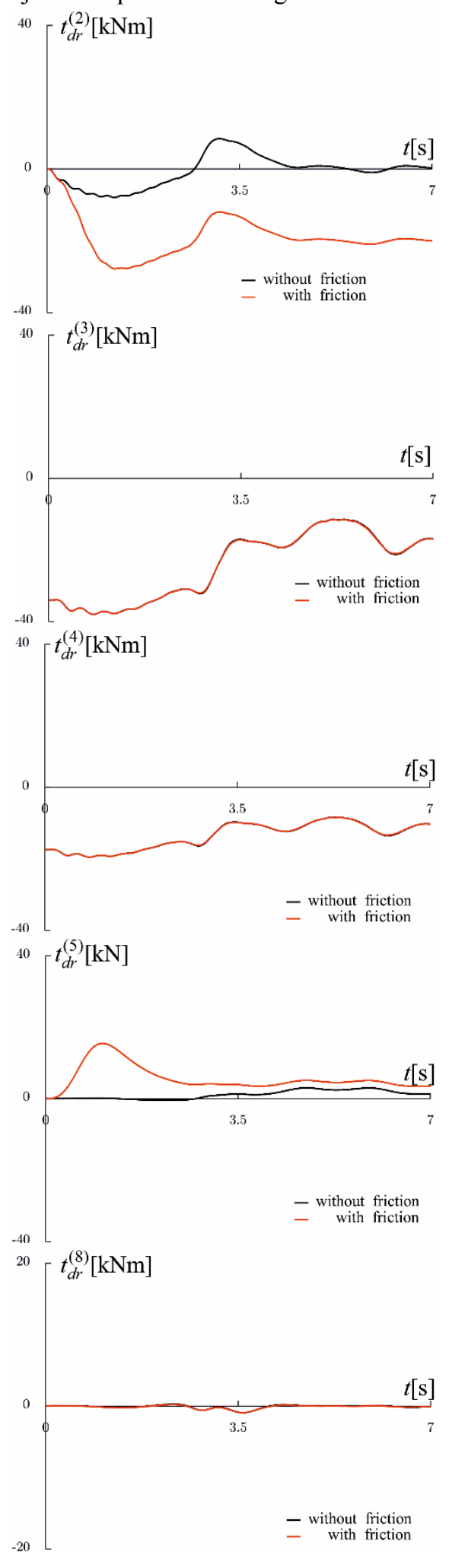

Fig. 4. Courses of driving torques and force.

After analysing the results, a significant influence can be noticed of the friction on the driving torque in joint 2 and the driving force in joint 5 . These influences are not significant in the other drives.

The trajectories of selected points of load determined in $\hat{\mathbf{x}}^{(0)} \hat{\mathbf{y}}^{(0)}$ are presented in Fig. 5.

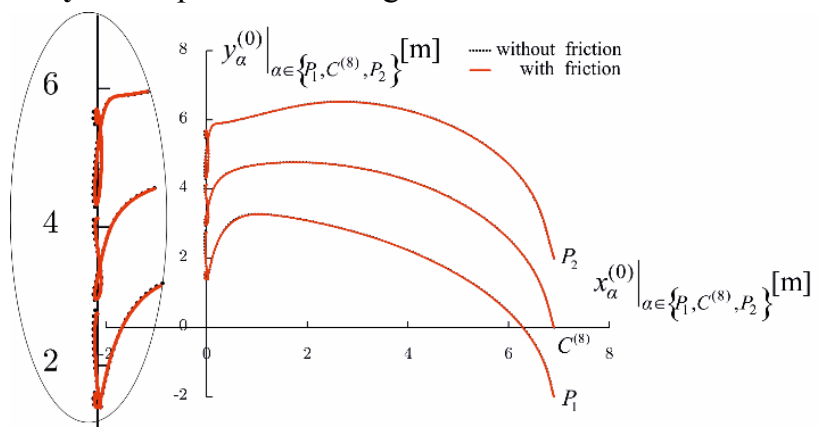

Fig. 5. Trajectories of selected points of the load.

An analysis of the trajectories allows to observe that friction does not significantly affect the behaviour of the load.

\section{Conclusions}

The dynamics analysis of a grab crane is presented in the paper. In formulating the mathematical model of the crane, the following are taken into account: flexibility of the supports, drives and friction in the joints, which is modelled using the Dahl friction model. The influence of these quantities on the driving torques and force, and also the behaviour of the load, are analysed. In the author's opinion, the model presented here can be useful in the process of design.

\section{References}

1. E. Papadopoulos, S. Sarkar, On the dynamic modeling of an articulated electrohydraulic forestry machine in Proc. of the 1996 AIAA Forum on Advanced Developments in Space Robotics, WI, 1-2 August 1996.

2. A. Urbaś, Analysis of flexibility of the support and its influence on dynamics of the grab crane, Latin American Journal of Solids and Structures 10(1) (2013).

3. B. Posiadała, P. Waryś, D. Cekus, M. Tomala, The dynamics of the forest crane during the load carrying, International Journal of Structural Stability and 13(7)

(2013). doi: $10.1142 / \mathrm{S} 0219455413400130$

4. P. La Hera and D. O. Morales, Model-based development of control systems for forestry cranes, Journal of Control and Science and Engineering, vol. 2015, no. ID 256951, doi:10.1155/2015/256951.

5. J. J. Craig, Introduction to robotics. Mechanics and control. Addison-Wesley Publishing Company, Inc., (1989)

6. P. R. Dahl, Solid friction damping of mechanical vibrations. AIAA Journal, 14(12) (1976)

7. E. I. Jurevič (ed.), Dynamics of robot control, Nauka, Moscow (1984), in Russian 